\title{
Frictional and mechanical behavior of graphene/UHMWPE composite coatings
}

\author{
A. Chih ${ }^{1}$, A. Ansón-Casaos ${ }^{2}$, J.A. Puértolas ${ }^{1 *}$ \\ ${ }^{1}$ Department of Materials Science and Technology, Instituto de Investigación en \\ Ingeniería de Aragón, I3A, Universidad de Zaragoza, 50018 Zaragoza, Spain \\ ${ }^{2}$ Instituto de Carboquímica ICB-CSIC, Miguel Luesma Castán 4, 50018 Zaragoza, Spain
}

*Correspondence to Prof. José A. Puértolas

Department of Materials Science and Technology,

Escuela de Ingeniería y Arquitectura-I3A, Universidad de Zaragoza,

E-50018, Zaragoza, Spain

Tel. : +34 976762521

Fax. : +34 976761957

e-mail: japr@unizar.es 


\begin{abstract}
Bulk-modified ultra-high molecular weight polyethylene (UHMWPE) is a biomaterial currently used for joint replacements. As an alternative, we assess the frictional behavior of coatings based on graphene/UHMWPE composites and sprayed graphene, which maintain the high performance of the UHMWPE matrix. Composite coatings were prepared with 0-4.6 wt.\% of graphene nanoplatelets (GNP) and 1-2 layered graphene (2LG). Mechanical properties of the coatings were measured by nanoidentation and friction coefficients were determined with a ball-on-disk tribometer. The results indicate that GNP present better tribological behavior than 2LG. Spraycoated GNP shows the lowest friction, 40\% less than UHMWPE, although with low adhesion. An increase of $10 \%$ in elastic modulus and $30 \%$ in hardness was measured for composite coatings with $2-5$ wt.\% graphene.
\end{abstract}

KEYWORDS: Polyethylene; graphene; coating; friction coefficient 


\section{INTRODUCTION}

Ultra-high molecular weight polyethylene (UHMWPE) has been one of the most relevant materials for total hip and knee replacements during the last 50 years [1]. Current research is focused on improving its wear resistance, chemical stability and mechanical properties. The first is necessary to reduce the UHMWPE debris generated in the bearing contact, which ultimately lead to osteolysis and the subsequent prosthesis revision. Chemical stability is essential to reduce the "in vivo" degradation process caused by the eventual appearance of free radicals on the material. Lastly, an improvement in mechanical properties would allow the redesign of total hip replacements with larger femoral heads, decreasing the risk of dislocation and fracture. First and second generations of highly cross-linked polyethylenes (HXLPEs) are bulk-modified materials, which try to address this former paradigm, even though losing some of the intrinsic properties of virgin UHMWPE.

The addition of functional fillers to polymers is a common strategy to improve their performance in terms of mechanical, electrical, biocompatibility and tribological properties. The filler composition, size, concentration, orientation and shape are specific for each involved property and polymeric matrix. In 1970, UHMWPE was reinforced with carbon fiber (Poly II®). After that, many attempts have been made with other reinforcement materials, including the new nanostructured carbons such as carbon nanotubes and more recently graphene related materials [2].

The use of graphene as a filler in UHMWPE is related to its intrinsic stiffness and mechanical resistance, given by a high elastic modulus of $1 \mathrm{TPa}$ and an ultimate tensile stress of $130 \mathrm{GPa}$. On the other hand, the great surface area of graphene should provide extended interfacial interactions with the matrix, increasing the load transfer and strength of the composites. Concerning the chemical stability, the presence of a network of 
conjugated double bonds provides a large electron donor-acceptor capacity with the ability to easily react with free radicals [3]. Finally, the two-dimensional character of graphene points out its potential as a high-performance solid lubricant and additive in liquid lubricants [4].

As a consequence of those outstanding properties, several works in the literature have assessed the influence of graphene fillers on the tribological and mechanical properties of different polymers including polyacrylonitrile [5], polyether ether ketone (PEEK) [6, 7], polyvinyl chloride [8], polyimide (PI) [9], and polytetrafluoroehylene (PTFE) [10].

The goal of this work is evaluating the frictional behavior of UHMWPE coated with either a graphene/UHMWPE composite or a sprayed graphene layer. The UHMWPE composite layer provides a better adhesion to the polymer matrix than other previously proposed coatings made of different materials. Moreover, the surface modification of UHMWPE keeps the mechanical performance of the polymer matrix, while currently used crosslinked polyethylenes, prepared under electron beam or gamma irradiation, suffer a negative variation in certain mechanical properties, most specifically in toughness. On the other hand, this kind of UHMWPE coatings incorporates the outstanding lubrication capability of graphene, which could reduce the friction and increase the surface hardness. The second effect can positively contribute to reduce the wear rate and consequently the particle debris. In this preliminary work, we evaluate the influence of graphene percentages, the graphene type and the deposition method on the friction coefficient and mechanical properties of the coatings.

\section{MATERIALS AND METHODS}

\section{Materials}


Medical grade UHMWPE powder, reference GUR1050 ${ }^{\circledR}$, was supplied by Celanese. Two different graphene materials were used as fillers, both supplied by Avanzare, Spain. One of them was a multilayer nanoplatelet graphene (GNP), reference avanPLAT-40 ${ }^{\circledR}$, which is obtained by mechanical exfoliation of graphite. It has a lateral platelet size of $40 \mu \mathrm{m}$ and an average thickness around $10 \mathrm{~nm}$, and each particle consists of $\leq 30$ layers. The other graphene material, reference avanGRAPHENE ${ }^{\circledR}$, is a 1-2 layered graphene (2LG) with a lateral platelet size of $20 \mu \mathrm{m}$ x $20 \mu \mathrm{m}$, which is obtained by a modified Hummers method.

\section{Composite consolidation}

The graphene powders were mixed with UHMWPE particles in a ball mill for 8 hours at $400 \mathrm{rpm}$ to obtain a homogeneous dispersion of the fillers in the polymer matrix. Different mixtures with GNP concentrations of $0,0.47,1.42,2.34$ and 4.58 wt.\% in the coating were prepared and the samples are accordingly denoted as G\%/PE (Table 1). The powder mixtures were incorporated onto the surface of a pre-formed UHMWPE disk, previously compressed under $15 \mathrm{MPa}$ at room temperature. In this way, graphene content was of 0 , $0.1,0.3,0.5$ and $1 \mathrm{wt} . \%$ in the total final disk $(\mathrm{d}=90 \mathrm{~mm}, \mathrm{t}=4 \mathrm{~mm})$. The composite layer and the substrate were thermo-compressed together for 30 minutes in a hot platen press (Specac, Slough, UK) at $155{ }^{\circ} \mathrm{C}$ and $15 \mathrm{MPa}$. In order to study the influence of compression temperature on the tribological properties, a composite coating with 4.58 wt.\% GNP was pressed at $240{ }^{\circ} \mathrm{C}(\mathrm{G} / \mathrm{PE}-\mathrm{HT})$. On the other hand, to assess the influence of the graphene type, another composite coating was consolidated with $4.58 \mathrm{wt} . \%$ of the $1-2$ layered graphene (2LG/PE).

\section{Spray-painting coating}

One sample (G-SP) was performed by air-spraying a suspension of $50 \mathrm{mg}$ of GNP in 50 mg of absolute ethanol onto the surface of a pre-formed UHMWPE disk. The suspension was prepared through mixing in an ultrasound bath for $5 \mathrm{~min}$. The solid remained in 
suspension for long enough to perform the spray-coating process, as the settling time was of 30-60 min. The spray-coating process was followed by thermo-compression at $155^{\circ} \mathrm{C}$ and $15 \mathrm{MPa}$. All the samples studied in the present work are listed in Table 1.

\section{Physicochemical characterization}

Scanning electron microscopy (SEM) images were taken from sample interior surfaces after cryogenic fracture. Fourier-transform infrared (IR) spectroscopy was performed in a Bruker Vertex 70 spectrometer. Consolidated surfaces were measured in an attenuated total reflectance (ATR) configuration, while powder graphene materials were mixed with spectroscopic $\mathrm{KBr}$, pressed to form pellets, and measured in a transmission configuration. Raman spectra were obtained by means of a HORIBA Jobin Yvon Raman spectrometer HR 800UV, using a $532 \mathrm{~nm}$ laser. Optical microscopy images $110 \times 140 \mu \mathrm{m}^{2}$ were taken using a 50x objective under a halogen lamp illumination. Electrical resistance on the surface of insulating specimens was measured between two tungsten needles separated by a distance of $2 \mathrm{~mm}$, using a Keithley 4200-SCS system. An in-line 4 point probe configuration with equidistant probe separations of $2.24 \mathrm{~mm}$ was utilized for the measurement of surface resistivity and electrical conductivity. Electrical conductivity of pure graphene materials was measured on pellets prepared by compression at $10 \mathrm{MPa}$.

\section{Coefficient of friction}

A commercial ball-on-disk tribometer (CSM instruments; Peseux, Switzerland) allowed the continuous monitoring of the friction coefficient. Samples were cut into disks with a diameter of $20 \mathrm{~mm}$ and a thickness of $3 \mathrm{~mm}$ to be inserted in the tribometer rotating vessel. The average roughness of the specimen surface was of $R_{a}=1.05 \pm 0.05 \mu \mathrm{m}$. The counterpart was an alumina ball, with a diameter of $6 \mathrm{~mm}$ and $\mathrm{R}_{\mathrm{a}}=0.050 \pm 0.002 \mu \mathrm{m}$. In order to reproduce physiological and biomechanical conditions the environment 
temperature was set to $37^{\circ} \mathrm{C}$ and the applied load was $5 \mathrm{~N}$, resulting in an initial contact pressure of $37 \mathrm{MPa}$, which is in the range of peak contact stress found for contemporary polyethylene (PE) tibia inserts. Two different radiuses for the circular track were used with each material: $4 \mathrm{~mm}$ with a sliding speed of $0.05 \mathrm{~m} / \mathrm{s}$ for 2 hours, and $1.5 \mathrm{~mm}$ at 0.02 $\mathrm{m} / \mathrm{s}$ for 5.2 hours. Deionized water was used as lubricant.

\section{Roughness and nanoindentation studies}

Average surface roughness was measured with a confocal microscope Sensofar PLm 230D (Sensofar, Barcelona, Spain). Nanoindentation assays were applied for hardness assessment and to evaluate changes in the mechanical properties compared to the pristine resin. In-depth hardness profiles were obtained from each material using a G200 nanoindenter (Agilent Tecnologies, Englewood, Colorado, USA) in the Continuous Stiffness Measurement (CSM) mode. The maximum depth programmed in the tests was $1500 \mathrm{~nm}$ and the indentation rate was close to $5 \mathrm{~nm} / \mathrm{s}$. Student's t-tests served to detect significant differences between the nanoidentation results of the different materials. A level of $p<0.05$ was selected as indicative of significance.

\section{RESULTS AND DISCUSSION}

\section{Surface morphology and physicochemical characterization}

Figure 1 shows SEM images of cryogenic fracture surfaces in two representative samples:

G4.6/PE and G-SP. The images correspond to a fast crack propagation that starts from a notch on the specimen side opposite to the coating according to the bending mode used to fracture the material. Figure 1.a encompasses the whole fracture surface for the specimen G4.6/PE at low magnification. Two clearly different zones are observed corresponding to the GNP/PE composite layer and the UHMWPE substrate. The pure PE area displays a 
smooth lumpy appearance similar to other previous PE fracture images captured by an instrumented double notch Izod test [11]. The GNP/PE composite region is of around 700 $\mu \mathrm{m}$, and the presence of the graphene platelets embedded into PE can be observed at high magnification (Figure 1.b). A certain level of agglomeration, in terms of graphene sheet stacking, can be deduced from the image, and could influence the composite mechanical properties [12]. The fracture surface of the G-SP sample at low magnification (Figure 1.c) presents a lumpy morphology like in the composite-coated PE. The spray-painted layer, with a thickness around 2-4 $\mu \mathrm{m}$, can be only observed in micrographs at higher magnifications (Figure 1.d).

In order to understand IR and Raman spectra of the coatings, the powder fillers and the bare matrix were measured. Figures 2.a-c show the results for GNP and 2LG graphene materials and the thermally compressed bare UHMWPE, respectively. The main IR features for $\mathrm{GNP}$ are the $\mathrm{C}=\mathrm{C}$ aromatic ring stretching at $1576 \mathrm{~cm}^{-1}[13]$ and the $\mathrm{O}-\mathrm{H}$ modes of adsorbed water at 1632 and $3443 \mathrm{~cm}^{-1}$. In addition, the 2LG powder shows a phenol C-O stretching vibration at $1217 \mathrm{~cm}^{-1}$ [13], indicating that $2 \mathrm{LG}$ contains a much larger number of oxygen chemical groups than GNP. The oxygen difference between both graphene materials arises from their different preparation methods. The IR spectrum for UHMWPE clearly shows the methylene $\mathrm{C}-\mathrm{H}$ asymmetric/symmetric stretching at $2918 / 2849 \mathrm{~cm}^{-1}$, methylene $\mathrm{C}-\mathrm{H}$ bending at $1464 \mathrm{~cm}^{-1}$, and methylene $-\left(\mathrm{CH}_{2}\right)_{\mathrm{n}}$ - rocking at $721 \mathrm{~cm}^{-1}$ [13]. The ATR-IR spectra of all the composite coatings, including the G-SP sample, are reminiscent of bare PE with the only insertion of weak graphene modes in the range of $1500-1650 \mathrm{~cm}^{-1}$. As an example, Figure 2 includes the spectrum for the G4.61/PE sample, which shows a weak feature at $1648 \mathrm{~cm}^{-1}$ with a shoulder at around $1576 \mathrm{~cm}^{-1}$ due to the graphene $\mathrm{C}=\mathrm{C}$ aromatic stretching.

Raman spectra of GNP and 2LG powder materials show the typical disorder-induced D band at around $1350 \mathrm{~cm}^{-1}$, and the tangential mode $\mathrm{G}$ band at around $1580 \mathrm{~cm}^{-1}$. 
Differences in the peak maximum positions and band widths can be assigned to the increased lattice stress and oxidation level of 2LG. Moreover, a clear difference appears in the G/D intensity ratio of both materials, with a value of $15.3 \pm 6.7$ in GNP and $1.0 \pm 0.4$ in 2LG. The second order 2D band appears at around $2700 \mathrm{~cm}^{-1}$, and G/2D intensity ratios for GNP and 2LG are respectively $2.7 \pm 0.1$ and $5.6 \pm 1.6$. The Raman spectrum of GNP is typical of mechanically exfoliated graphene, while the spectrum of 2LG well corresponds to a material prepared by liquid phase oxidation [14]. The decrease in the G/D and G/2D intensity ratios for $2 \mathrm{LG}$, compared to GNP, is associated to the introduction of lattice defects during the adapted Hummers method used for the exfoliation of graphite to obtain 2LG.The Raman spectrum of UHMWPE exhibits characteristic features that have been previously assigned [15]: a band associated with the $\mathrm{C}-\mathrm{C}$ stretching vibrational mode at $1131 \mathrm{~cm}^{-1}$, another band at $1295 \mathrm{~cm}^{-1}$ due to the $-\mathrm{CH}_{2}$ - twisting vibration, the $-\mathrm{CH}_{2}$ bending at $1441 \mathrm{~cm}^{-1}$, and two prominent stretching vibrations of the $-\mathrm{CH}_{2}$ - methylene group at 2849 and $2884 \mathrm{~cm}^{-1}$. Raman spectra of the composite coatings are an uneven superposition of both graphene and PE component features. Opposite to IR analysis, strongly resonant graphene bands often hide PE when micro-Raman point measurements are performed. As an example, a spectrum measured on the G4.6/PE coating is shown in Figure 2.

Graphene distribution on the composite surface was characterized by optical microscopy, since IR and Raman spectroscopies detect the presence of graphene, but are not capable of obtaining a quantitative estimate. Figure 3 shows three representative pictures for each sample coating. Under a yellow halogen lamp, graphene-enriched regions are observed in a yellowish light colour, while PE is dark brown. In the G\%/PE series, the area of light regions increases with the GNP percentage. According to the literature, graphene nanofillers are typically added to PE at concentrations in the range of $1 \mathrm{wt} \%$ [11]. In the present work, the graphene percentage is increased up to a $4.8 \mathrm{wt} \%$, which allows a nearly 
complete coverage of the PE exposed surface with the utilized mixing protocol. The images for the G4.6/PE and G/PE-HT materials, which were prepared with an identical nominal amount of GNP, are similar. The G-SP coating logically shows the lightest appearance, as all the graphene was painted on the PE surface instead of being mixed with PE powders. However, the 2LG/PE sample, in spite of having an identical amount of graphene than G4.6/PE, shows a darker colour, what could be related to different reflectance properties of $2 \mathrm{LG}$ compared to GNP.

Table 2 includes DC electrical characteristics for the coating specimens and their components. The surface resistance of $\mathrm{G} \% / \mathrm{PE}$ series and $2 \mathrm{LG} / \mathrm{PE}$ coatings was relatively close to that of PE, around $10^{14} \Omega$, while it underwent a strong decreased down to $6.6 \cdot 10^{3}$ $\Omega$ for the G/PE-HT coating. This behaviour could be related to a better graphene dispersion during the consolidation process at high temperature, due to the increase of the self-diffusion of molecular chains across the UHMWPE particle boundaries [16]. Electrical conductivity of the G-SP layer was high, although somewhat lower than that of pressed GNP powders.

\section{Coefficient of friction}

The experimental data of coefficient of friction (COF) versus the sliding distance are here discussed considering 4 separate variables: the graphene concentration in the GNP/UHMWPE composite coating, the graphene filler type, the consolidation temperature, and finally the graphene deposition method (powder mixing or spraypainting).

Figure 4 includes the COF vs distance curves for all the tested specimens at $r=4 \mathrm{~mm}$ (Figures $4 \mathrm{a}, 4 \mathrm{~b}$ and $4 \mathrm{c}$ ) and $\mathrm{r}=1.5 \mathrm{~mm}$ (Figures $4 \mathrm{c}, 4 \mathrm{~d}$ and $4 \mathrm{e}$ ). The G\%/PE composites show a short running period followed by a nearly stationary value, both at $r=4 \mathrm{~mm}$ (Figures 4a) and $\mathrm{r}=1.5 \mathrm{~mm}$ (Figure 4d). Measurements for $\mathrm{r}=1.5 \mathrm{~mm}$ present a 
somewhat higher data dispersion, indicating that the surface local heterogeneity might be more apparent than for $r=4 \mathrm{~mm}$. In the present work, the steady COF of UHMWPE was found to be around 0.05-0.06 (Figure 4.a and 4d), which is lower than the value of 0.08, at $4000 \mathrm{~m}$ of sliding distance, previously reported by Puertolas et al. [17]. However, in that previous test, the lubricant was bovine serum, while in the present work the disk was immersed in deionized water, being identical the other experimental parameters. Table 3 indicates the average COFs in the whole testing time. As a general trend, the frictional outcomes indicate that the addition of graphene usually increases the coefficient of friction. However, at the highest graphene loadings (G2.3/PE and G4.6/PE specimens) and $\mathrm{r}=4 \mathrm{~mm}$, the average COF values are below the raw UHMWPE without significant statistical differences. The last trend was more clearly observed by Lahiri et al. [18] in a nano-scratch test, in which the progressive incorporation of GNP in PE up to 1 wt.\% caused a decrease in COF during scratching. However, such a trend has not been observed for other graphene-based fillers in UHMWPE; in fact, the presence of graphene oxide (GO) nanosheets caused an increase in the initial frictional coefficient instead of reducing it [19].

In other polymer matrices different from $\mathrm{PE}$, different results are also found in the literature regarding the effect of graphene on the COF. A $15 \%$ reduction was detected in PVC with 2 wt.\% of multi-layered graphene [8], and a $20 \%$ reduction was measured in PEEK with 1 wt.\% of GO [6]. The positive tribological influence of graphene fillers is in accordance with molecular dynamic simulation findings, which predict a strong decrease in the COF against the sliding of an iron nanorod [20]. However, an increase in the COF was reported upon the addition of 2 wt.\% of graphene nanopowder to PEEK [7]. Some attempts to reduce COF via an enhancement of the interfacial interaction filler-matrix provided different results: chemically-modified reduced graphene oxide (RGO) 
significantly improved the COF of PAN [5] while fluorinated graphene did not affect it [9].

The COF for the 2LG/PE specimen reached an average value around 0.08 , being much higher than the COF for G4.6/PE (Figures 4.b and 4e). This macroscopic result is in line with a tribological behaviour recently described at the nanoscale through atomic force microscopy [21, 22] and molecular dynamic simulations [23, 24]. Those studies clearly pointed to a layer-dependent friction behaviour, so that a reduced number of layers provokes a higher friction than a multilayer system. The classical interpretation of this effect is based on the interlayer sliding which is higher in multilayer graphene than in fewlayered materials. However, new mechanisms have been recently introduced, as the outof-plane elastic deformation generated on the graphene sheets, which is higher in thinner sheets. This mechanism provides a high resistance to the indenter movement and consequently higher friction. As far as we know, only Bhargava et al. [10] have previously studied the influence of the filler platelet thickness on the macroscopic tribological effects. In fact, frictional outcomes for PTFE composites indicated that the influence of platelet thickness is relevant at graphene contents above $1.1 \mathrm{wt} . \%$, and the COF decreased substantially when the filler thickness changed from $1.25 \mathrm{~nm}$ to $60 \mathrm{~nm}$.

The lowest COF was obtained in the G-SP specimen (Figure 4.c). The COF reaches a plateau at values close to 0.03 at $r=4 \mathrm{~mm}$ and 0.05 at $\mathrm{r}=1.5 \mathrm{~mm}$. However, this value remains constant only in a short term, up to $30 \mathrm{~m}$ in the best specimen. From this point the friction underwent an abrupt change to values close to 0.12 . This behaviour is a consequence of the fracture of the thin graphene layer implanted by spray painting, which could provoke the appearance of a third body effect and increased the COF up to values even higher than for UHMWPE. In another G-SP specimen, the qualitative behaviour is similar, although with a shorter distance at the lowest value of 0.05 . Regarding the G/PEHT material, the high temperature consolidation process does not improve the tribological 
behaviour of the composites, although affects positively to the electrical behaviour, as we have previously mentioned, and to the mechanical properties, as we will discuss later.

\section{Nanoindentation measurements}

All the nano-modulus profiles showed an analogous qualitative behaviour regardless the coating type. In Figure 5.a, we show representative results for the raw UHMWPE, G4.6/PE and 2LG/PE materials. The profiles present a start-up value followed by a rapid decrease until reaching an approximately constant value. The initial and final values for UHMWPE were 1.41 GPa and 1.08 GPa respectively. In order to compare the results for all the materials, a mean value corresponding to the whole in-depth profile was calculated and represented in Figure 5.b. Composite coatings with GNP fillers provide a maximum increase in modulus of $10 \%$ for nanofiller weight percentages in the range of 1.4-4.6 wt.\%, while the effect $2 \mathrm{LG}$ is negligible. This behaviour is reminiscent of previous literature works with other graphene-based composites at similar filler loadings, including GO [25], RGO [12] and graphene nanosheet (GNS) composites [26]. In the reports with GO and RGO materials the modulus remained nearly constant, while in GNS composites the increase was around $12 \%$, thus similar to that in our GNP composites. Therefore, stiffness outcomes are far from those expected taking into account the exceptional modulus of graphene and the great surface area of GNP, which should provide an increased number of interfaces with the matrix, improving load transfer. The limited improvement in the modulus has to be associated with a segregated structure of the filler around the UHMWPE powders, generated during the consolidation process by particle agglomeration.

The temperature change during hot-pressing does not induce any significant change in the composite stiffness. Moreover, the temperature increase provides a modification in the electrical network (Table 2) without any appreciable changes in the modulus, in agreement 
with our previous measurements on RGO/UHMWPE composites [12]. Therefore, the increase observed in the modulus compared to the raw UHMWPE would be associated to the presence of $4.6 \mathrm{wt} \%$ of GNP rather than to the influence of the consolidation temperature. Finally, a strong reduction in the modulus by $30 \%$ was measured in the G-SP sample due to the low adhesion of the spray-painted pure GNP layer, which has been also reflected in the friction coefficient behaviour.

The low adhesion of the graphene layer can be intuitively associated to the lack of strong enough specific interactions between graphene and PE units. It has been demonstrated that van der Waals interactions cannot support the strong adhesion of graphene to substrates such as amorphous $\mathrm{SiO}_{2}$ [27]. Experimental studies and molecular dynamics simulations suggest an improvement in the interfacial interactions between graphene and polyethylene for biomedical applications through the chemical functionalization of graphene with amines [28-30]. Therefore, chemical functionalization would be a way for improving the G-SP sample properties. In addition, modifications in the preparation method would be possible, including an optimization of the GNP layer thickness, different strategies for the improvement of the GNP ink quality, and a careful study of the processing parameters.

Concerning hardness, nano-indentation provided an in-depth profile similar to the elastic modulus (Figure 6.a). In UHMWPE the value was of $0.05 \mathrm{GPa}$ at depths of indentation above $600 \mathrm{~nm}$, according with previously published values $[17,31]$. The profiles for the composite coatings show similar trends, with higher initial values than PE and achieving a nearly identical final value at higher depths, above $150 \mathrm{~nm}$.

Figure 6.b compares mean hardness values corresponding to the whole in-depth profile for all the studied specimens. The present findings point to a positive influence of GNP fillers on hardness, since above 1.4 wt.\% it significantly $(p \leq 0,0001)$ increased with respect to virgin PE. A maximum increase close to $32 \%$ was reached for $2.3 \mathrm{wt} \% \mathrm{GNP}$, and it is kept for the G/PE-HT composite coating, consolidated at $240{ }^{\circ} \mathrm{C}$ instead of $175^{\circ} \mathrm{C}$. When 
hardness of G4.6/PE is compared to 2LG/PE in order to assess the influence of graphene type, the results indicate that the number of graphene layers is not relevant for hardness. Finally, the G-SP coating showed a decrease in hardness, with a significantly lower value than PE, confirming the weak adhesion between the GNP material and the substrate.

It can be stated that graphene nanoparticles with percentages of $2-5$ wt. $\%$ in PE are able to improve hardness from 67 to nearly $90 \mathrm{MPa}$. A similar maximum value is also reached when the composite is consolidated at high temperature (G/PE-HT sample). The effect appears to be related to the high surface reflectance (Figure 3) and electrical conductivity (Table 2), which could be due to a greater amount of graphene at the surface after consolidation a $240{ }^{\circ} \mathrm{C}$, leading to a positive effect on hardness. The improvement is similar to that reached by He ion implantation [31], and one order of magnitude lower than the diamond-like-carbon (DLC) coating effect on UHMWPE substrates [17]. However, the present method is the most efficient in terms of costs and the time required for the surface modification process, and it avoids limitations due to the DLC layer low adhesion. The improved hardness of GNP/UHMWPE coatings could be a suitable way to reduce the adhesion wear observed in friction tests at short times, since according to Archard equation, an increase in the hardness of the softest contacting surfaces would decrease the volume of wear debris. The study of the wear behaviour, the optimization of the composite layer thickness and the introduction of chemical specific interactions between graphene and PE should be considered for future works.

\section{CONCLUSIONS}

The tribological properties of UHMWPE can be improved through coating techniques based on graphene materials and graphene/PE composites. A GNP/PE composite cover decreases the friction coefficient by a $10 \%$. The tribological properties of 
graphene/PE composites depend on the graphene type: results with the multi-layered GNP material were better than with 2-layer graphene. A spray-painted pure GNP layer decreases the friction coefficient by a 40-50\%, but the layer adhesion and hardness need to be improved. Surface modulus and hardness can be respectively improved by a $10 \%$ and a $+30 \%$ through the addition of a $2-5$ wt. $\%$ GNP. Surface electrical resistivity of graphene/PE coatings can be decreased through an increase in the consolidation temperature from 175 to $245^{\circ} \mathrm{C}$, without modifying tribological and surface mechanical properties.

\section{ACKNOWLEDGEMENTS}

Special thanks are directed to the Analysis Service of Instituto de Carboquímica, ICBCSIC, in particular to M.D. Domínguez, N. Fernández and I. Fernández. This work has been funded by MINECO and European Regional Development Fund (ENE 2016-79282C5-1-R), and Government of Aragon and European Social Fund (DGA-ESF-T66 "Grupo Consolidado"). 
FIGURE 1. Cryogenic fracture SEM micrographs of the G4.6/PE composite at magnifications of a) $25 x$ and b) $500 x$, and the G-SP coating at magnifications of c) $25 x$ and d) 1500x.

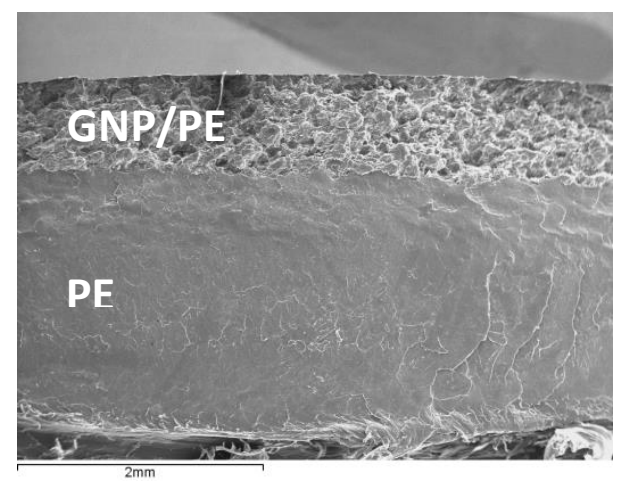

(a)
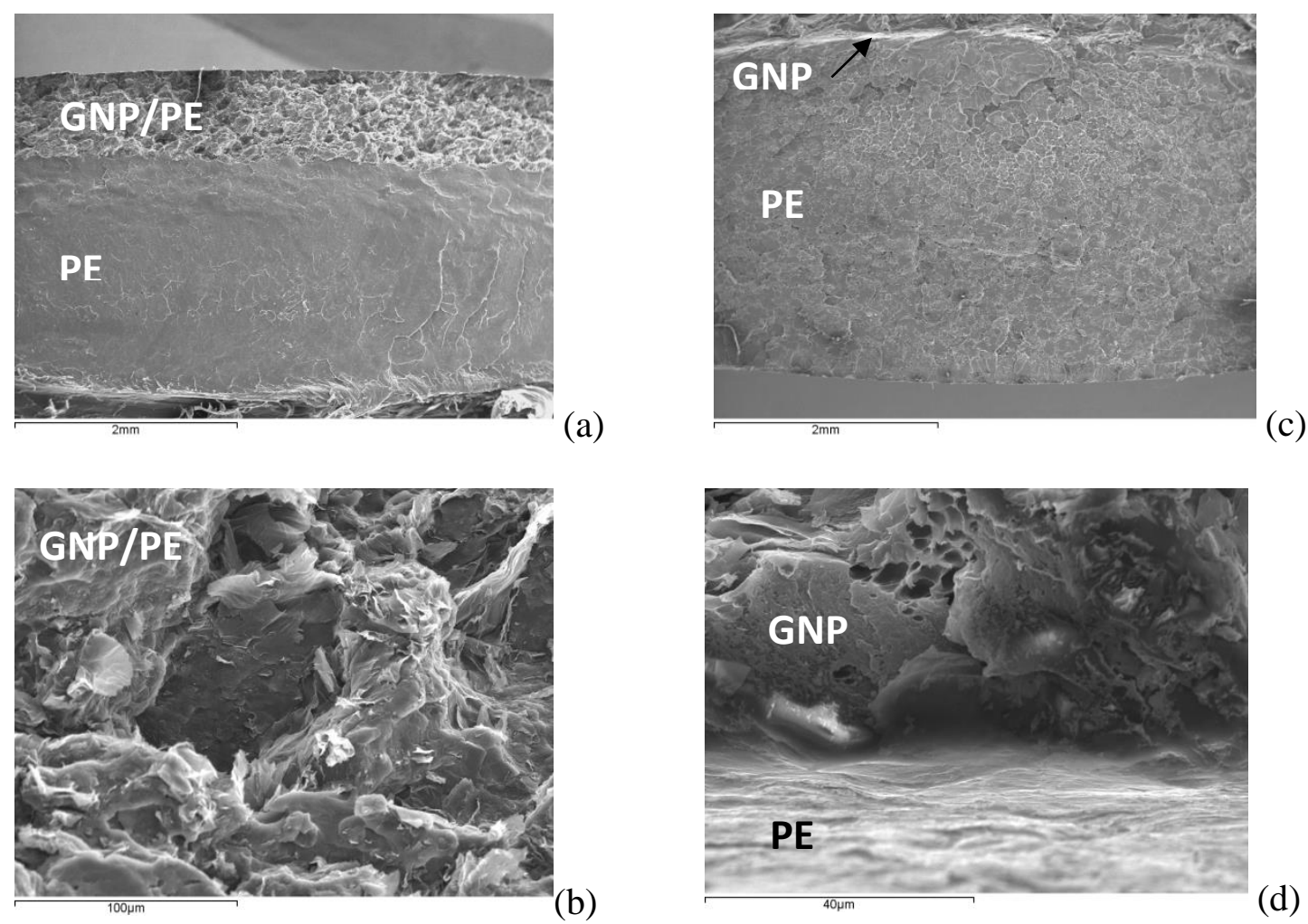
FIGURE 2. Normalized baseline-subtracted IR (left) and Raman (right) spectra of a) GNP, b) 2LG, c) UHMWPE, and d) G4.6/PE coating.
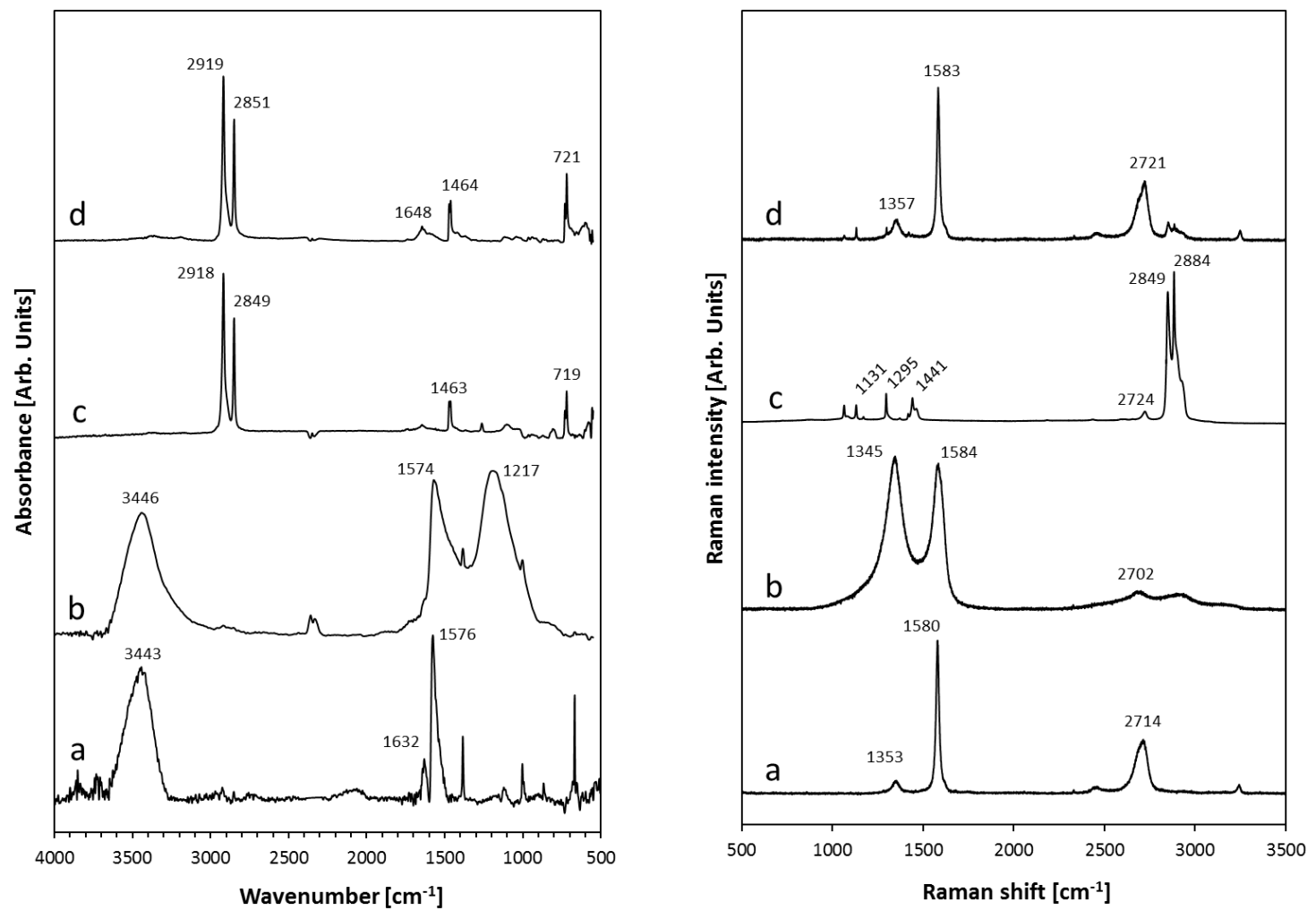
FIGURE 3. Optical micrographs under a halogen lamp illumination (real size is $140 \mathrm{x}$ $110 \mu \mathrm{m})$ for the different coating surfaces.

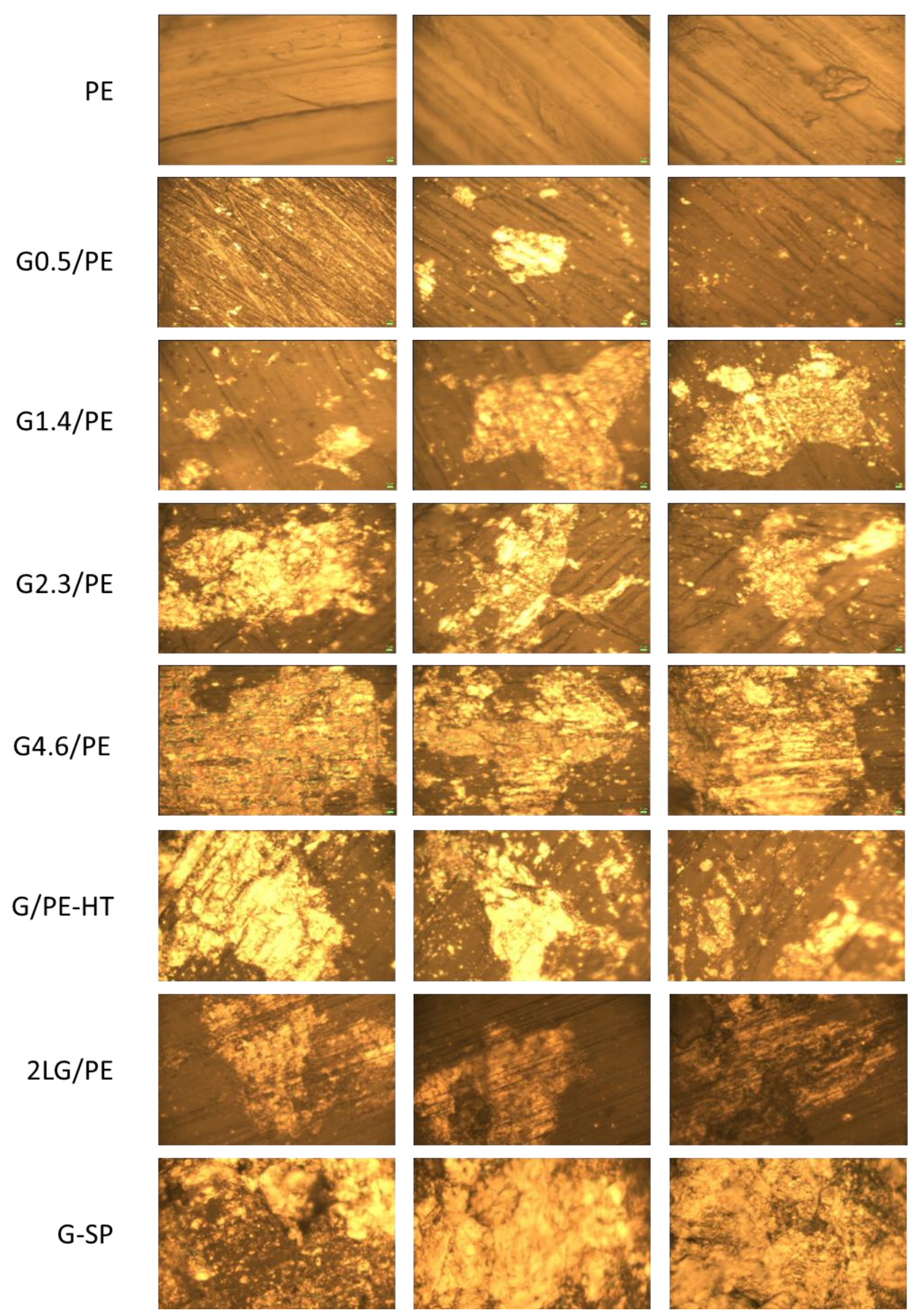


FIGURE 4. Plots of the frictional coefficient vs. sliding distance for: a) G\%/PE coatings at $\mathrm{r}=4 \mathrm{~mm}, \mathrm{~b}$ ) G4.6/PE, 2LG/PE and G/PE-HT at $4 \mathrm{~mm}, \mathrm{c}$ ) G-SP at $\mathrm{r}=4 \mathrm{~mm}$ and $1.5 \mathrm{~mm}$, d) $\mathrm{G} \% / \mathrm{PE}$ coatings at $\mathrm{r}=1.5 \mathrm{~mm}, \mathrm{e}) \mathrm{G} 4.6 / \mathrm{PE}$ and $2 \mathrm{LG} / \mathrm{PE}$ at $1.5 \mathrm{~mm}$.
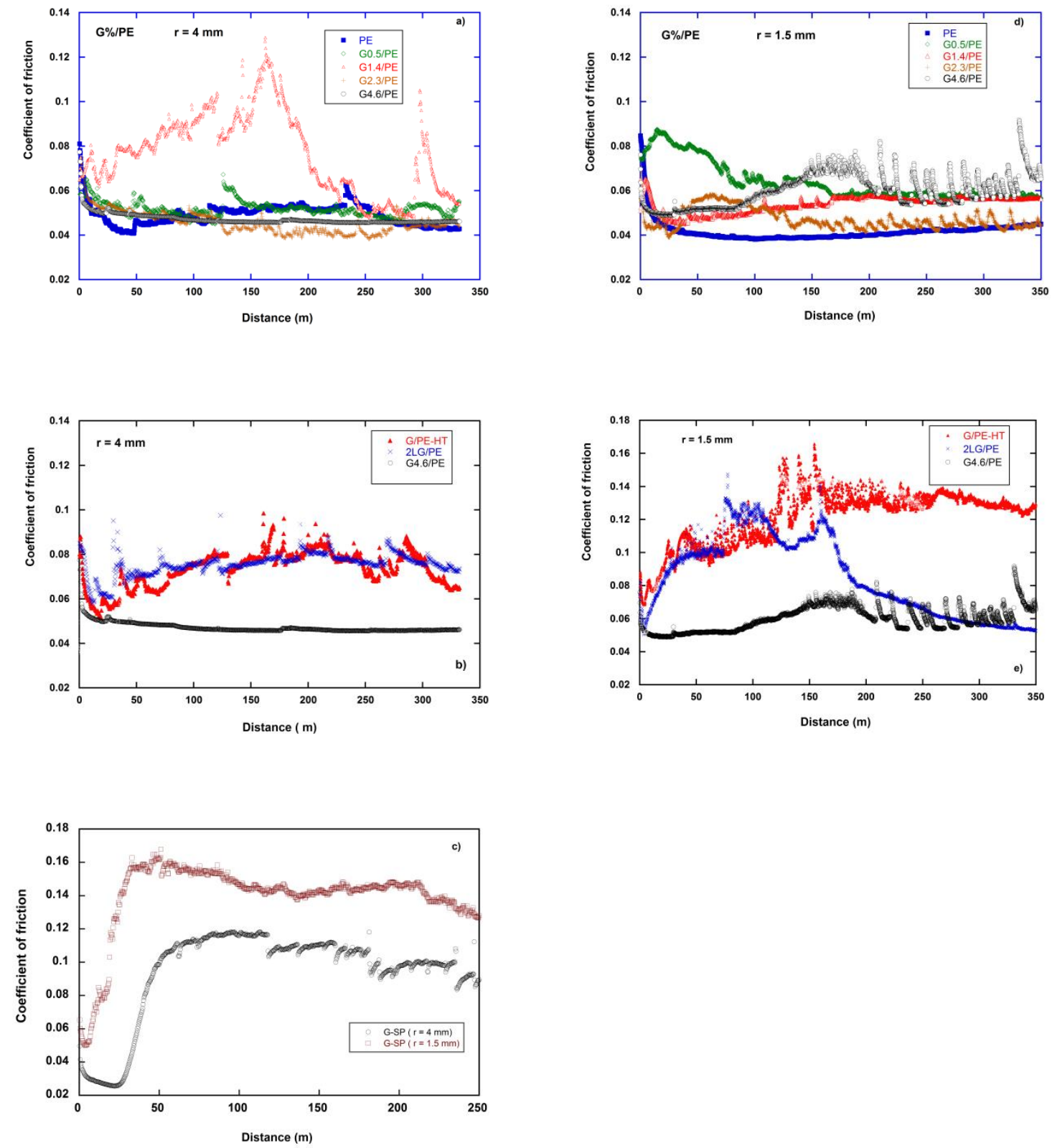
FIGURE 5. a) Modulus in-depth profiles for raw UHMWPE and the composites coatings with 4.6 wt.\% GNP and 4.6 wt.\% 2LG materials; b) Mean values calculated from the whole in-depth profile of all the composites coatings. (*) Statistically significant differences $(p<0.0001)$ compared to raw UHMWPE .

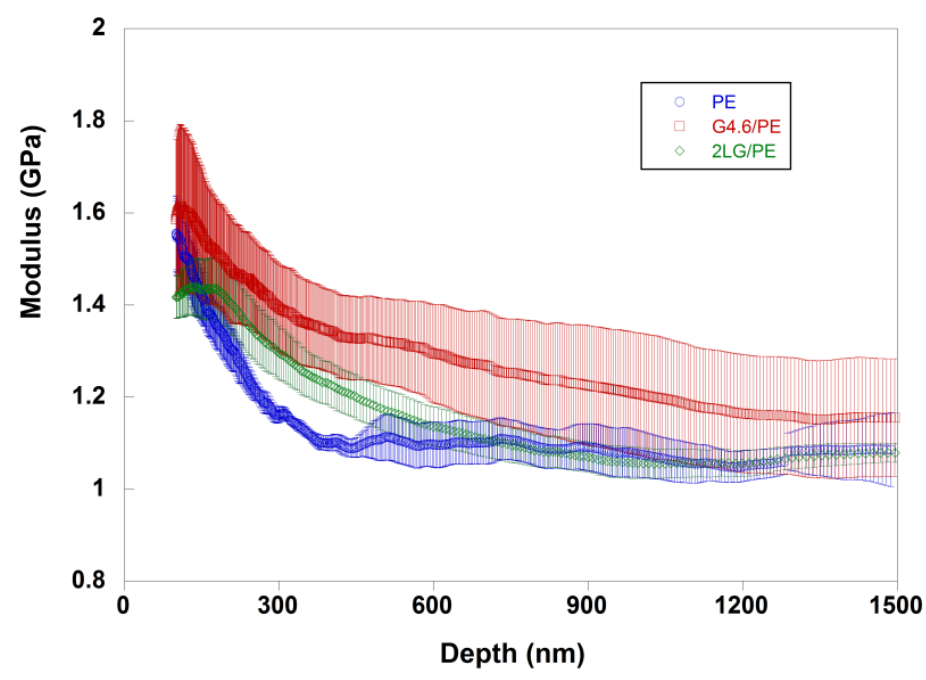

(a)

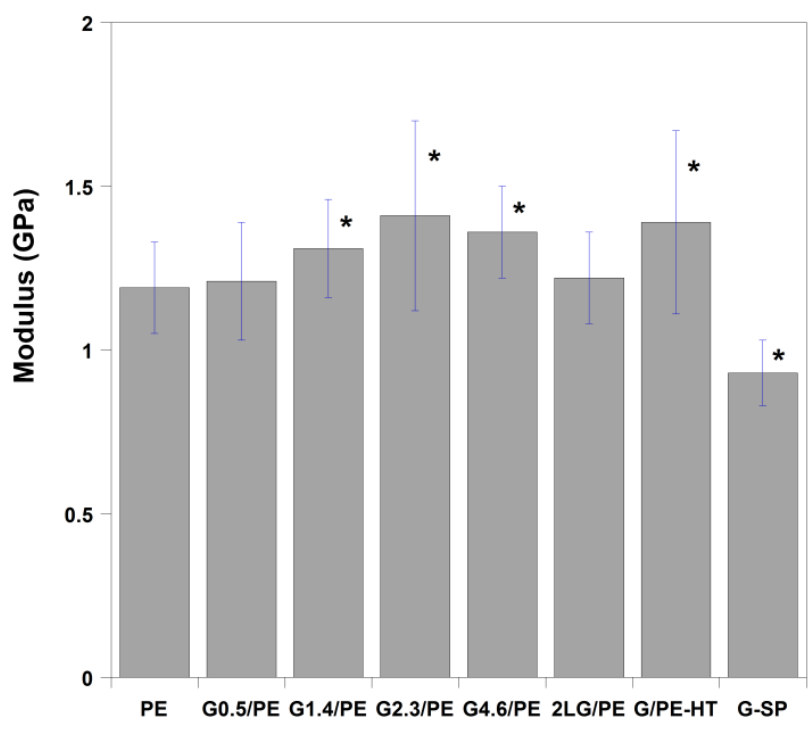

(b) 
FIGURE 6. a) Hardness in-depth profiles for raw UHMWPE and the composites coatings with 4.6 wt.\% GNP and 4.6 wt.\% 2LG materials; b) Mean values calculated from the whole in-depth profile of all the composites coatings. (*) Statistically significant differences $(p<0.0001)$ compared to raw UHMWPE.

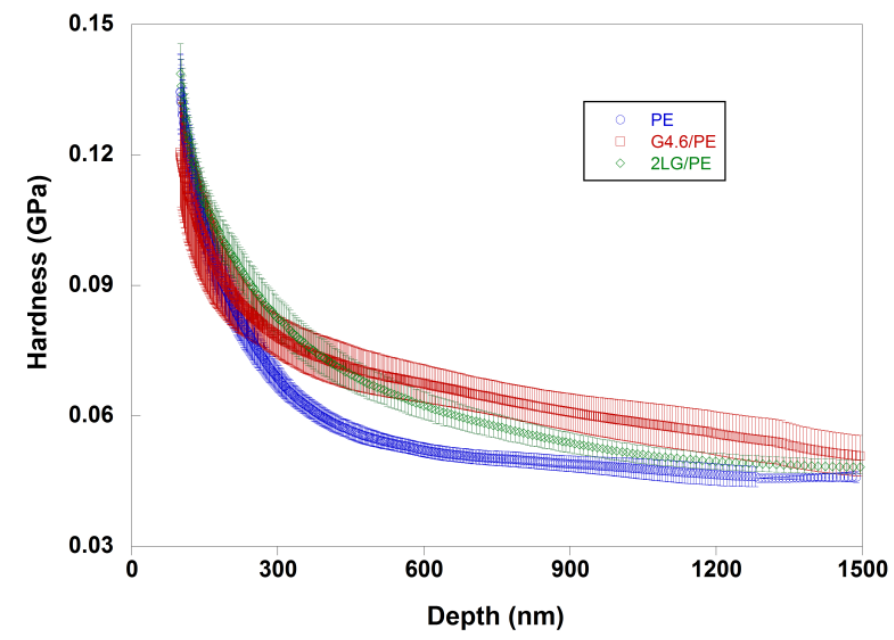

(a)

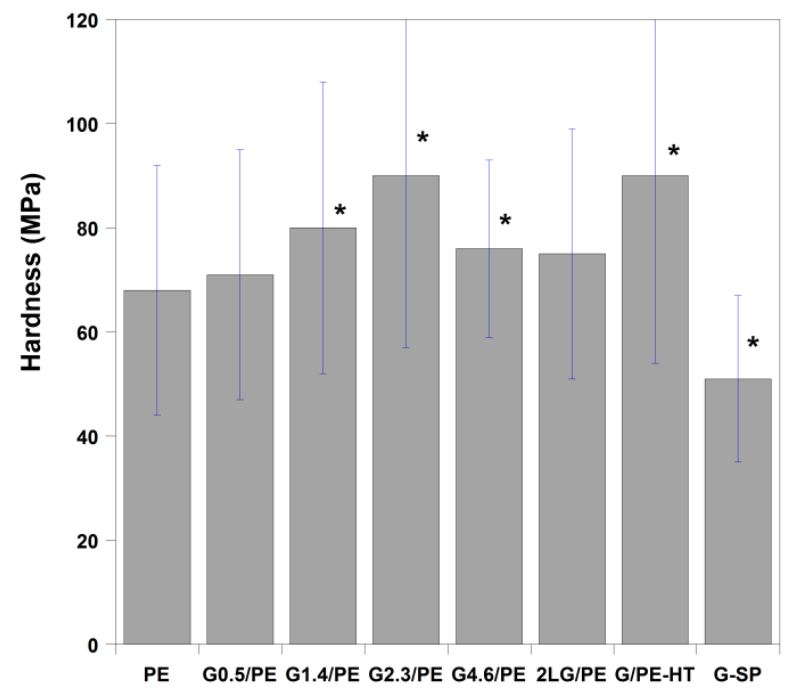

(b) 
Table 1. Composition parameters of the coatings on UHMWPE studied in the present work.

\begin{tabular}{|l|l|l|l|l|l|}
\hline Coating & Matrix & Graphene type $^{a}$ & Loading [wt.\%] & $T\left[^{\circ} \mathrm{C}^{b}\right.$ & Method \\
\hline G\%/PE & GUR 1050 & GNP & $0.5,1.4,2.3,4.6$ & 175 & Powder mixing \\
\hline G/PE-HT & GUR1050 & GNP & 4.6 & 240 & Powder mixing \\
\hline 2LG/PE & GUR 1050 & 2LG & 4.6 & 175 & Powder mixing \\
\hline G-SP & --- & GNP & --- & 175 & Spray-painting \\
\hline
\end{tabular}

${ }^{\mathrm{a}} \mathrm{GNP}$ : graphene nanoplatelets < 30 layers; 2LG: 1-2 layer graphene

${ }^{\mathrm{b} C o n s o l i d a t i o n ~ t e m p e r a t u r e ~}$

Table 2. Electrical characterization of components and coating specimens.

\begin{tabular}{|l|l|l|l|}
\hline Sample & $R[\Omega]^{a}$ & $R_{s}[\Omega / s q .]^{b}$ & $\sigma\left[S \cdot \mathrm{cm}^{-1}\right]^{c}$ \\
\hline UHMWPE & $8.83 \cdot 10^{14}$ & --- & --- \\
\hline G4.6/PE & $2.59 \cdot 10^{14}$ & --- & --- \\
\hline 2LG/PE & $7.69 \cdot 10^{13}$ & --- & --- \\
\hline G/PE-HT & $6.62 \cdot 10^{3}$ & $1.91 \cdot 10^{3}$ & $1.81 \cdot 10^{-2}$ \\
\hline G-SP & --- & 74.9 & 45.7 \\
\hline 2LG & --- & $1.20 \cdot 10^{-1}$ & 66.8 \\
\hline GNP & --- & $1.87 \cdot 10^{-2}$ & $9.40 \cdot 10^{2}$ \\
\hline
\end{tabular}

${ }^{a} R: 2$ point probe surface resistance (probe separation $=2 \mathrm{~mm}$ )

${ }^{\mathrm{b}} \mathrm{R}_{\mathrm{s}}: 4$ collinear point probe surface resistivity $(\mathrm{s}=2.2 \mathrm{~mm})$

${ }^{c} \sigma: 4$ point probe bulk conductivity 
Table 3. Average of the coefficient of friction obtained with the ball-on-disk tribometer at two different circular track radiuses.

\begin{tabular}{|l|l|l|}
\hline Material & $r=4 \mathrm{~mm}$ & $r=1.5 \mathrm{~mm}$ \\
\hline $\mathrm{PE}$ & $0.049 \pm 0,005$ & $0.042 \pm 0.005$ \\
\hline G0.5/PE & $0.052 \pm 0.004$ & $0.064 \pm 0.008$ \\
\hline G1.4/PE & $0.074 \pm 0.018$ & $0.054 \pm 0.004$ \\
\hline G2.3/PE & $0.046 \pm 0.005$ & $0.047 \pm 0.005$ \\
\hline G4.6/PE & $0.047 \pm 0.003$ & $0.062 \pm 0.008$ \\
\hline G/PE-HT & $0.073 \pm 0.008$ & $0.121 \pm 0.017$ \\
\hline 2LG/PE & $0.075 \pm 0.005$ & $0.084 \pm 0.024$ \\
\hline
\end{tabular}




\section{REFERENCES}

[1] Kurtz SM. The UHMWPE Biomaterials Handbook. 3rd Edition . Elsevier. 2016.

[2] Puertolas JA, Kurtz SM. Evaluation of carbon nanotubes and graphene as reinforcements for UHMWPE-based composites in arthroplastic applications: A review. Journal of the mechanical behavior of biomedical materials. 2014;39:129-45.

[3] Denis PA. Density Functional Investigation of Thioepoxidated and Thiolated Graphene. Journal of Physical Chemistry C. 2009;113:5612-9.

[4] Berman D, Erdemir A, Sumant AV. Graphene: a new emerging lubricant. Mater Today. 2014;17:31-42.

[5] Mo YF, Yang ML, Lu ZX, Huang FC. Preparation and tribological performance of chemically-modified reduced graphene oxide/polyacrylonitrile composites. Compos Part a-Appl S. 2013;54:153-8.

[6] Song HJ, Li N, Li YJ, Min CY, Wang Z. Preparation and tribological properties of graphene/poly(ether ether ketone) nanocomposites. Journal of Materials Science. 2012;47:6436-43.

[7] Kalin M, Zalaznik M, Novak S. Wear and friction behaviour of poly-ether-etherketone (PEEK) filled with graphene, WS2 and CNT nanoparticles. Wear. 2015;332:85562.

[8] Wang H, Xie GY, Zhu ZG, Ying Z, Zeng Y. Enhanced tribological performance of the multi-layer graphene filled poly(vinyl chloride) composites. Compos Part a-Appl S. 2014;67:268-73.

[9] Ye XY, Liu XH, Yang ZG, Wang ZF, Wang HG, Wang JQ, et al. Tribological properties of fluorinated graphene reinforced polyimide composite coatings under different lubricated conditions. Compos Part a-Appl S. 2016;81:282-8.

[10] Bhargava S, Koratkar N, Blanchet TA. Effect of Platelet Thickness on Wear of Graphene-Polytetrafluoroethylene (PTFE) Composites. Tribol Lett. 2015;59.

[11] Puertolas JA, Pascual FJ, Martinez-Morlanes MJ. Impact resistance and fractography in ultra high molecular weight polyethylenes. Journal of the mechanical behavior of biomedical materials. 2014;30:111-22.

[12] Pascual J. Tenacidad en polietilenos de ultra alto peso molecular altamente reticulados y en materiales compuestos de esa matriz polimérica reforzada con grafeno. Tesis Universidad de Zaragoza. 2016.

[13] Coates J. Interpretation of infrared spectra, a practical approach. Chichester: John Wiley and Sons Ltd; 2000.

[14] Valles C, Nunez JD, Benito AM, Maser WK. Flexible conductive graphene paper obtained by direct and gentle annealing of graphene oxide paper. Carbon. 2012;50:835-44. [15] Taddei P, Affatato S, Fagnano C, Bordini B, Tinti A, Toni A. Vibrational spectroscopy of ultra-high molecular weight polyethylene hip prostheses: influence of the sterilisation method on crystallinity and surface oxidation. J Mol Struct. 2002;613:121-9. [16] Fu J, Ghali BW, Lozynsky AJ, Oral E, Muratoglu OK. Ultra high molecular weight polyethylene with improved plasticity and toughness by high temperature melting. Polymer. 2010;51:2721-31.

[17] Puertolas JA, Martinez-Nogues V, Martinez-Morlanes MJ, Mariscal MD, Medel FJ, Lopez-Santos C, et al. Improved wear performance of ultra high molecular weight polyethylene coated with hydrogenated diamond like carbon. Wear. 2010;269:458-65. [18] Lahiri D, Hec F, Thiesse M, Durygind A, Zhang C, Agarwal A. Nanotribological behavior of graphene nanoplatelet reinforced ultra high molecular weight polyethylene composites. Tribology International. 2014;70:165-9.

[19] Tai ZX, Chen YF, An YF, Yan XB, Xue QJ. Tribological Behavior of UHMWPE Reinforced with Graphene Oxide Nanosheets. Tribol Lett. 2012;46:55-63. 
[20] Li YL, Wang SJ, Wang Q. A molecular dynamics simulation study on enhancement of mechanical and tribological properties of polymer composites by introduction of graphene. Carbon. 2017;111:538-45.

[21] Lee C, Li QY, Kalb W, Liu XZ, Berger H, Carpick RW, et al. Frictional Characteristics of Atomically Thin Sheets. Science. 2010;328:76-80.

[22] Kawai S, Benassi A, Gnecco E, Sode H, Pawlak R, Feng XL, et al. Superlubricity of graphene nanoribbons on gold surfaces. Science. 2016;351:957-61.

[23] Kim HJ, Seo KJ, Kim DE. Investigation of Mechanical Behavior of Single- and Multi-Layer Graphene by using Molecular Dynamics Simulation. Int J Precis Eng Man. 2016;17:1693-701.

[24] Li SZ, Li QY, Carpick RW, Gumbsch P, Liu XZ, Ding XD, et al. The evolving quality of frictional contact with graphene. Nature. 2016;539:541-+.

[25] Chen YF, Qi YY, Tai ZX, Yan XB, Zhu FL, Xue QJ. Preparation, mechanical properties and biocompatibility of graphene oxide/ultrahigh molecular weight polyethylene composites. European Polymer Journal. 2012;48:1026-33.

[26] Ren PG, Di YY, Zhang Q, Li L, Pang H, Li ZM. Composites of Ultrahigh-MolecularWeight Polyethylene with Graphene Sheets and/or MWCNTs with Segregated Network Structure: Preparation and Properties. Macromol Mater Eng. 2012;297:437-43.

[27] Kumar S, Parks D, Kamrin K. Mechanistic Origin of the Ultrastrong Adhesion between Graphene and a-SiO2: Beyond van der Waals. Acs Nano. 2016;10:6552-62. [28] Nikkhah SJ, Moghbeli MR, Hashemianzadeh SM. Investigation of the interface between polyethylene and functionalized graphene: A computer simulation study. Curr Appl Phys. 2015;15:1188-99.

[29] Upadhyay R, Naskar S, Bhaskar N, Bose S, Basu B. Modulation of Protein Adsorption and Cell Proliferation on Polyethylene Immobilized Graphene Oxide Reinforced HDPE Bionanocomposites. Acs Applied Materials \& Interfaces. 2016;8:11954-68.

[30] Hui J, Ren PG, Sun ZF, Ren F, Xu L, Zhang ZP, et al. Influences of interfacial adhesion on gas barrier property of functionalized graphene oxide/ultra-high-molecularweight polyethylene composites with segregated structure. Compos Interface.

2017;24:729-41.

[31] Rodriguez RJ, Medrano A, Garcia JA, Fuentes GG, Martinez R, Puertolas JA. Improvement of surface mechanical propel-ties of polymers by helium ion implantation. Surf Coat Tech. 2007;201:8146-9. 